\title{
Article \\ Phytochemical Profile of Eight Categories of Functional Edible Oils: A Metabolomic Approach Based on Chromatography Coupled with Mass Spectrometry
}

\author{
Carmen Socaciu ${ }^{1, *(D)}$, Francisc Dulf ${ }^{2}$, Sonia Socaci ${ }^{1}{ }^{(D}$, Floricuta Ranga ${ }^{1}$, Andrea Bunea ${ }^{3}$, Florinela Fetea ${ }^{1}$ and \\ Adela Pintea ${ }^{4}$ (D)
}

check for

updates

Citation: Socaciu, C.; Dulf, F.; Socaci,

S.; Ranga, F.; Bunea, A.; Fetea, F.;

Pintea, A. Phytochemical Profile of

Eight Categories of Functional Edible

Oils: A Metabolomic Approach Based

on Chromatography Coupled with

Mass Spectrometry. Appl. Sci. 2022,

12, 1933. https://doi.org/10.3390/

app12041933

Academic Editor: Agata Górska

Received: 11 January 2022

Accepted: 10 February 2022

Published: 12 February 2022

Publisher's Note: MDPI stays neutral with regard to jurisdictional claims in published maps and institutional affiliations.

Copyright: () 2022 by the authors Licensee MDPI, Basel, Switzerland. This article is an open access article distributed under the terms and conditions of the Creative Commons Attribution (CC BY) license (https:/ / creativecommons.org/licenses/by/ $4.0 /)$.
1 Department of Food Science, Faculty of Food Science and Technology, University of Agricultural Sciences and Veterinary Medicine Cluj-Napoca, 400372 Cluj-Napoca, Romania; sonia.socaci@usamvcluj.ro (S.S.); floricuta.ranga@usamvcluj.ro (F.R.); florinelafetea@usamvcluj.ro (F.F.)

2 Faculty of Agriculture, University of Agricultural Sciences and Veterinary Medicine Cluj-Napoca, 400372 Cluj-Napoca, Romania; francisc.dulf@usamvcluj.ro

3 Faculty of Animal Science and Biotechnology, University of Agricultural Sciences and Veterinary Medicine Cluj-Napoca, 400372 Cluj-Napoca, Romania; andrea.bunea@usamvcluj.ro

4 Faculty of Veterinary Medicine, University of Agricultural Sciences and Veterinary Medicine Cluj-Napoca, 400372 Cluj-Napoca, Romania; apintea@usamvcluj.ro

* Correspondence: carmen.socaciu@usamvcluj.ro

Featured Application: The data presented here recommend a systematic analytical flow based on a metabolomic approach to be applied for different foods, food bioresources or byproducts valorization, identifying the profile of valuable components, including phytochemicals, as authenticity biomarkers and health-promoting molecules.

\begin{abstract}
Functional vegetable oils are highly considered not only for their nutritional value, but also for their health benefits. The profile of phytochemicals responsible for their quality is useful also for the identification of possible mislabeling or adulteration. The comparative composition of eight categories (sunflower, pumpkin, hempseed, linseed, soybean, walnut, sea buckthorn and olive) of commercial vs. authentic oils was determined. Fatty acids, volatiles, carotenoids, tocopherols, and phenolic components were analyzed by gas- and liquid chromatography-based techniques coupled with diode array, mass spectrometry, or fluorescence detection. Classification models, commonly used in metabolomics, e.g., principal component analysis, partial least squares discriminant analysis, hierarchical clusters and heatmaps have been applied to discriminate each category and individual samples. Carotenoids, tocopherols, and phenolics contributed mostly, qualitatively, and quantitatively to the discrimination between the eight categories of oils, as well as between the authentic and the commercial ones. This metabolomic approach can be easily implemented and the heatmaps can be considered as "identity" cards of each oil category and the quality of commercial oils, comparative to the authentic ones of the same botanical and geographical origin.
\end{abstract}

Keywords: edible functional oils; food identity; phytochemicals' profile; gas- and liquid chromatography; mass spectrometry; chemometrics; metabolomics

\section{Introduction}

Vegetable oils are essential food products of high nutritional value and a major agrifood commodity subjected to adulteration before commercialization. Consumers are appreciating oils' nutritional potential but are concerned about their quality and safety, while the food producers are careful to their quality, influenced by multiple environmental and technological factors affecting mostly the fatty acids, such as oxidation and higher levels of trans derivatives [1-3]. Over the years, the Codex standards for fats and oils have been modified to enhance the supervision of vegetable oils quality and authenticity, and 
available databases and international standards are evolving (https://www.fao.org/3/ t4660t/t4660t0e.htm accessed 15 January 2021). The EU legislation by Regulation (EEC) 2568/91 established methods for olive oil analysis, but not for other types of vegetable oils. Meanwhile, in the context of the fight against food fraud, the publication of EU regulation $2017 / 625$ focused on legal regulations related to edible oils. Authenticity is a very important quality criterion, considering the significant differences of composition, safety, and price between the cold-pressed (extra virgin, virgin) and refined oils, as well the mislabeling of commercial products. Moreover, along with olive oils, other edible oils are considered functional or specialty (e.g., pumpkin, walnut, soybean, hemp, linseed, sea buckthorn), since, beside the nutritional function, they contain bioactive compounds with health benefits and reduced risk of some diseases, mainly cardiovascular and neurovegetative, due to their high amounts of essential polyunsaturated lipids rich in linoleic acid and linolenic fatty acids, phospholipids, phytosterols, and bioactive phenolics [4].

Edible vegetable oils contain mainly triacylglycerols (up to 98\%) and different phytochemicals (micronutrients) as tocopherols, phytosterols and carotenoids, essential components for our diet, having high nutritional value. The most consumed edible oils in Central and Eastern Europe are sunflower, soybean, rapeseed oils, but also increasingly olive, pumpkin, grapeseed and palm oils $[5,6]$. Most publications related to the identity (quality and authenticity) of oils are dominated by studies on different olive oil varieties and commercial quality. The health benefits of olive oil are well documented, being mainly attributed to their high content in monounsaturated fatty acids (MUFAs), e.g., oleic and palmitoleic acids, but also to sterols, carotenoids, tocopherols and polyphenolic compounds (tyrosol derivatives and oleuropein), all being known for their antioxidant activity [7-13]. These natural antioxidants are found in many other functional oils, but fewer data were available regarding their composition and identity, dependent on their botanical phenotype and geographical distribution. Metabolomics is an advanced technology which offers a comprehensive characterization of small, bioactive molecules (phytochemicals) which play a central role in oils' identity. Analytical platforms such as gas chromatography and liquid chromatography coupled with diode array, fluorescence or mass spectrometry-detection techniques (GC-MS and LC-DAD-RF-MS) and nuclear magnetic resonance (NMR) spectroscopy are essential prerequisites used to ensure a systematic study about the quality and authenticity biomarkers of vegetable oils. Mainly, chromatography is applied for the separation and mass spectrometry for identification. The GC-MS is a standardized method to identify and quantify fatty acids, volatile components, and sterols, while LC-DAD-RF-MS or fluorescence (HPLC-RF) are useful for the separation, identification and quantification of carotenoids, tocopherols and polyphenols ([14-22]. Meanwhile, infrared spectrometry also brings added value information, if subsequent multivariate classification methods are applied, e.g., by principal component analysis (PCA), partial least squares-discriminant analysis (PLS-DA) or cluster analysis (CA) [23-25]. Actually, any analysis needs an adequate chemometric evaluation by multivariate analysis, for improving the classification performance, particularly for challenging classification tasks such as the discrimination of edible oils of different biological or geographical origins, and genuine vs. adulterated commercial products [26-28]. The data fusion based on these complementary analytical techniques brings new knowledge and allows the identification of specific oil biomarkers (e.g., fatty acids, sterols and tocopherols) to ensure correct classification. Metabolomics concepts are very useful for real progress in the multivariate classifications, by different sophisticated statistical or machine learning methods $[8,10,12,29]$. The objective of this study was to compare the phytochemicals' profile of 30 edible oils from eight categories, based on their botanical origins (sunflower, pumpkin, hempseed, linseed, soybean, sea buckthorn and olive oil), to discriminate their specific identity biomarkers. There were four advanced techniques applied-GC-MS, HPLC-DAD, HPLC-RF and LC-ESI+MS-to separate and identify five different components (fatty acids, volatiles, tocopherols, carotenoids, and phenolic derivatives) from each vegetable oil (genuine vs. commercial) and to provide the value of qualitative and quantitative biomarkers for their recognition and authenticity. 
The chemometric analysis included a variety of classification models, including principal component analysis (PCA), partial least square discriminant analysis and hierarchical clustering by heatmaps. The differences among oils have been described and the specific recognition biomarkers were identified, creating an "identity card" for each category of oil.

\section{Materials and Methods}

\subsection{Oil Samples}

Eight oil categories, including 30 edible oils produced and commercialized in Romania (excepting olive oils) were investigated. From each category, one sample was authentic, process-controlled, and carefully supervised, as provided from trusted manufacturers. The commercial samples were purchased from retailers being labelled as "cold-pressed" "extra virgin", or "virgin" for olive oils. Table 1 contains data about their botanical origin, the declared/labelled quality grade, and sample codes which were used in the classification studies. The last column shows the codes of authentic samples.

Table 1. The botanical origin, the labelled quality, and codes used in all investigations and statistical classifications of commercial and authentic oils.

\begin{tabular}{ccc}
\hline Botanical Origin/Group nr. & Commercial Samples: Labelled Quality/Sample Code & Authentic Samples Code \\
\hline Sunflower (I) & Cold-pressed/SFO1, SFO2, SFO3 & $\mathrm{aSFO4}$ \\
Pumpkin (II) & Cold-pressed/PO1, PO2, PO3 & $\mathrm{aPO} 4$ \\
Linseed (III) & Cold-pressed/LSO1, LSO2 & $\mathrm{aLSO3}$ \\
Hempseed (IV) & Cold-pressed/HO1, HO2, HO3 & $\mathrm{aHO} 4$ \\
Soybean (V) & Cold-pressed/SO2, SO3 & $\mathrm{aSO} 1$ \\
Walnut (VI) & Cold-pressed/WO1, WO2, WO3 & $\mathrm{aWO4}$ \\
Sea buckthorn (VII) & Cold-pressed/SBO2, SBO3, SBO4 & $\mathrm{aSBO1}$ \\
Olive (VIII) & Extra virgin Italy (EVOO1), virgin Spain (EVOO2), Extra & - \\
\hline
\end{tabular}

Three aliquots of $50 \mathrm{~mL}$ from each oil were used for analysis and the measurements were done in triplicate from each aliquot.

\subsection{UV-VIS Spectrometry}

As a preliminary investigation, a volume of $10 \mathrm{~mL}$ from each oil was mixed with $10 \mathrm{~mL}$ hexane, vortexed for $3 \mathrm{~min}$, centrifuged and filtered through 0.25 microns membrane. The UV-VIS spectra were recorded using a Jasco V 530 double beam spectrometer, in the region $200-700 \mathrm{~nm}$, the maximum absorption being registered for each sample.

\subsection{Fatty Acid Analysis by GC-MS}

The GC-MS analysis of fatty acids was done by the standard transesterification procedure followed by separation of ethyl esters (FAMEs) using a PerkinElmer Clarus 600 T GCMS (PerkinElmer, Inc., Shelton, CT, DOOR) with a column Supelcowax $10(60 \mathrm{~m} \times 0.25 \mathrm{~mm}$ i.d., $0.25 \mu \mathrm{m}$ thickness (Supelco Inc., Bellefonte, PA, USA). Temperature program: initial temperature $140{ }^{\circ} \mathrm{C}$, then increase to $220^{\circ} \mathrm{C}$ by $7^{\circ} \mathrm{C} / \mathrm{min}$, the final temperature remained constant for $23 \mathrm{~min}$ up to the end. Injector temperature: $210^{\circ} \mathrm{C}$, split 1:24. Injected volume: $0.5 \mu \mathrm{L}$. Eluent: $\mathrm{He}, 0.8 \mathrm{~mL} / \mathrm{min}$. MS conditions: ionization energy $70 \mathrm{eV}$, trap current $100 \mu \mathrm{A}$, source temperature $150{ }^{\circ} \mathrm{C}$. Mass scanning range $m / z$ : 22-395, with $0.14 \mathrm{scan} / \mathrm{s}$. The identification of fatty acids was based on retention times, by comparison with retention times of fatty acids from a standards mixture (37 component FAME Mix, SUPELCO\# $47885-\mathrm{U}$ ) and by comparing the mass spectra with those from the MS database (NIST MS Search 2.0). Individual fatty acid levels were expressed in \% of area of the total identified fatty acid peak areas. 


\subsection{Volatiles' Profile by ITEX/GC-MS}

The extraction followed by separation of volatile compounds was performed using the "in-tube extraction" ITEX/GC-MS technique applied on a GC-MS Shimadzu QP2010 (Shimadzu Scientific Instruments, Kyoto, Japan) equipped with AOC-5000 CombiPAL autosampler (CTC Analytics, Zwingen, Switzerland) and ZB-5ms capillary column, $30 \mathrm{~m} \times 0.25 \mathrm{~mm}$ id $\times 0.25 \mu \mathrm{m}$ (film thickness) (Phenomenex, Torrance USA). A volume of $0.1 \mathrm{~mL}$ oil was placed in the headspace vial, sealed, and incubated at $60^{\circ} \mathrm{C}$ for $20 \mathrm{~min}$ with continuous stirring. The volatile compounds accumulated in the headspace of the ampoule were adsorbed, using a syringe, into a Tenax fiber (ITEX-2TRAPTXTA, (G23)-Siliconert 2000, Tenax 80/100 mesh, Switzerland) and then thermally desorbed in the GC injector. After each analysis, the fibre was cleaned by passing a warm stream of Nitrogen. The temperature program for the chromatographic column for the separation of volatile compounds was as follows: maintenance $2 \mathrm{~min}$ at $50{ }^{\circ} \mathrm{C}$, followed by an increase at $160{ }^{\circ} \mathrm{C}$ by $4{ }^{\circ} \mathrm{C} / \mathrm{min}$ and then an increase at $255^{\circ} \mathrm{C}$ by $15^{\circ} \mathrm{C} / \mathrm{min}$ and maintenance at this temperature for $10 \mathrm{~min}$. The carrier gas was helium, at a constant flow rate of $1 \mathrm{~mL} / \mathrm{min}$. The injector temperature, ion source and interface have been set to $250{ }^{\circ} \mathrm{C}$. The MS detector used the electronic impact ionization (EI) mode in a scanning range of $40-350 \mathrm{~m} / \mathrm{z}$. The split ratio was 1:20. Separated volatiles were identified by comparing the mass spectra with those from NIST27 and NIST147 mass spectrum libraries and verified by comparison with retention indices extracted from www.pherobase.com, accession 12 December 2021) and www.flavornet.org, accessed 10 December 2021) (for single-phase columns stationary like ZB-5ms). The results are expressed as a percentage of the total peak area.

\subsection{Carotenoid Profile and Quantification by HPLC-DAD}

Aliquots of $5 \mathrm{~g}$ from each oil were transferred to brown round bottom flasks and dissolved in $20 \mathrm{~mL}$ diethyl ether. Saponification was performed by stirring with $25 \mathrm{~mL}$ solution of $\mathrm{KOH}(30 \%)$ for $5 \mathrm{~h}$, in the dark, at room temperature, under inert gas. The reaction mixture was transferred into a separation funnel containing diethyl ether and water. The organic phase was washed repeatedly with $\mathrm{NaCl} 5 \%$ solution, until complete removal of alkali (neutral $\mathrm{pH}$ of the water phase). The organic phase was filtered and dried over sodium sulphate and the solvent was removed completely using a rotatory evaporator, at $35^{\circ} \mathrm{C}$ under reduced pressure. The residues containing carotenoids were dissolved in ethyl acetate and filtered through $0.2 \mu \mathrm{m}$ PTFE filters prior to injection.

HPLC separation of carotenoids was performed using a Shimadzu LC20 AT HPLC system (Shimadzu Corporation, Kyoto, Japan) with a SPDM20A diode array detector and a YMC C30 reversed phase column $(250 \times 4.6 \mathrm{~mm}$ i.d., $5 \mu \mathrm{m}$ particle size). The mobile phase consisted of solvent $A$, methanol/methyl tert-butyl ether/water (83:15:2, $v / v / v)$, and solvent $B$, methyl tert-butyl ether/methanol/water (80:7:2, v/v/v). A gradient system was used, as follows: 0 min $0 \%$ solvent $\mathrm{B}, 20 \mathrm{~min} 0 \% \mathrm{~B}, 130 \mathrm{~min} 82 \% \mathrm{~B}, 132 \mathrm{~min} 0 \% \mathrm{~B}$, followed by equilibration of column for $10 \mathrm{~min}$. The flow rate was $0.8 \mathrm{~mL} / \mathrm{min}$ and the chromatograms were recorded at $450 \mathrm{~nm}$. The DAD operated in the range of $300-600 \mathrm{~nm}$ for the acquisition of UV-VIS spectra. Carotenoid standards $\beta$-carotene, lutein and zeaxanthin (purity $\geq 98 \%, \geq 95 \%$ and $\geq 98 \%$, respectively) were purchased from Extrasynthese (Lyon, France). The identification of individual carotenoids was based on the comparison of their retention time, elution order on C30 column and UV-VIS spectra ( $\lambda$ max, spectral fine structure $(\% \mathrm{III} / \mathrm{II}))$ with those of the available standards and with literature data. The quantification was performed using external ten-points calibration curves constructed in the range 1-100 $\mu \mathrm{g} / \mathrm{mL}$ for the three major carotenoids. The correlation coefficients were $R^{2}=0.9912$ ( $\beta$-carotene), $R^{2}=0.9991$ (lutein) and $R^{2}=0.9996$ (zeaxanthin) [30].

\subsection{HPLC-RF Separation and Quantification of Tocopherols}

The oil samples $(0.5 \mathrm{~g})$ were dissolved in different volumes of hexane $(15-25 \mathrm{~mL})$, so that the tocopherol concentrations fell within the ranges of the calibration curves, filtered through $0.2 \mu \mathrm{m}$ Nylon filters and injected directly into the HPLC system (Shimadzu 
LC20 equipped with a Lichrosorb Si60 column $(250 \times 4.6 \mathrm{~mm}, 5 \mu \mathrm{m})$ and a fluorescence detector RF20A (excitation $\lambda=290 \mathrm{~nm}$ and emission $\lambda=330 \mathrm{~nm}$ ). The mobile phase was hexane: 2-propanol (99.5: $0.5, v / v)$, isocratically with a flow rate of $1 \mathrm{~mL} / \mathrm{min}$. Quantitative analysis was performed by external calibration with authentic standards ( $\alpha$-tocopherol, $\beta$-tocopherol, $\gamma$-tocopherol, and $\delta$-tocopherol) (Extrasynthese, Genay, France) in the concentration range of $0.25-50 \mu \mathrm{g} / \mathrm{mL}$, having correlation coefficients $R^{2}$ of 0.9980 .

\subsection{Extraction and Quantification of Total Phenolics}

To a volume of $3 \mathrm{~mL}$ oil, there was added first $3 \mathrm{~mL}$ hexane, and after vortex mixing $1 \mathrm{~min}, 5 \mathrm{~mL}$ of methanol: water (3:2) mixture was added and sonicated for $15 \mathrm{~min}$. Then, samples were centrifuged for $10 \mathrm{~min}$ at $3000 \mathrm{rpm}$. The upper phase was removed, and to the remaining methanol: water phase, $3 \mathrm{~mL}$ hexane was added. Finally, three washes with hexane were performed in order to discard the nonpolar lipids. The clean methanolic phase was evaporated on a rotary evaporator until dryness and taken up in $1 \mathrm{~mL}$ methanol 95\% for total polyphenols (TPs) determination using the Folin-Ciocalteu (FC) method [31]. Shortly, to $0.05 \mathrm{~mL}$ methanol extract, $2.35 \mathrm{~mL}$ water, $0.150 \mathrm{~mL}$ FC reagent and $0.450 \mathrm{~mL}$ natrium bicarbonate was added. In parallel for blank, the sample was replaced by methanol $95 \%$. After two hours in the dark, the samples' absorption at $750 \mathrm{~nm}$ was determined using a BioTek multidetection spectrometer (BioTek Instruments Inc., US). The calibration was done using gallic acid solutions from 0.0625 to $1 \mathrm{mg} / \mathrm{mL}$, the curve obtained was $y=0.9443 x+0.0608$. The $R^{2}$ value was 0.9945 . The concentration of total phenolics (TP-FC) was expressed in gallic acid equivalents (GAE), namely in $\mathrm{mg}$ GAE/100 mL oil.

\subsection{LC-ESI+-MS Identification and HPLC-DAD Quantification of Individual Phenolics}

Volumes of $0.3 \mathrm{~mL}$ from each methanolic extract, prepared as described above, were injected in an HPLC Agilent 1200 system equipped with a photodiode array detector (DAD) and coupled with a mass spectrometer (MS) single quadrupole Agilent model 6110 (Agilent Technologies, Santa Clara, CA, USA) was used for phenolics qualitative and quantitative analysis. The phenolics' separation was done on an Eclipse XDB C18 column $5 \mu \mathrm{m}(4.6 \times 150 \mathrm{~mm})$, (Agilent Technologies, Santa Clara, CA, USA) and a two mobilephase gradient was applied for $30 \mathrm{~min}, 25^{\circ} \mathrm{C}$, at a flow of $0.5 \mathrm{~mL} / \mathrm{min}$. Solvent A was $0.1 \%$ Ac acetic in water: $\mathrm{ACN}$ (99:1) and solvent $\mathrm{B}$ was $0.1 \%$ Ac acetic in acetonitrile. The gradient: $0 \mathrm{~min}, 5 \% \mathrm{~B}, 0-2 \mathrm{~min}, 5 \% \mathrm{~B}, 2-18 \mathrm{~min}, 5 \%-40 \%$ B, $18-20 \mathrm{~min}, 40 \%-90 \%$ B, $20-24 \mathrm{~min}, 90 \%$ B, $24-25 \mathrm{~min}, 90 \%-5 \% \mathrm{~B}, 25-30 \mathrm{~min}, 5 \% \mathrm{~B}$. For the accurate identification of each molecule, the MS used the ESI+ ionization, with a capillary voltage of $300 \mathrm{~V}$, at $300{ }^{\circ} \mathrm{C}$, nitrogen flow $7 \mathrm{~L} / \mathrm{min}$, for a $\mathrm{m} / z$ range from 100 to $1200 \mathrm{Da}$. The data acquisition and processing were done using the ChemStation Agilent software. For quantitative determinations, different calibration curves were done (Figure S1) using the LC-DAD system and different pure standards: gallic acid and vanillin (for hydroxybenzoic acid derivatives, recorded at $280 \mathrm{~nm}$ ), chlorogenic and ferulic acids (for hydroxycinnamic derivatives, recorded at $280 \mathrm{~nm}$ ), oleuropein (for tyrosols, recorded at $280 \mathrm{~nm}$ ), luteolin (for flavones, recorded at $340 \mathrm{~nm}$ ), quercetin (for flavonoids, recorded at $340 \mathrm{~nm}$ ) and pinoresinol (for lignans, recorded at $280 \mathrm{~nm}$ ). The total polyphenols (TP-LC) were calculated afterwards in each case from the sum of each phenolic concentration, considering the calibrations and each peak area in the LC-DAD.

\subsection{Multivariate Analysis}

All data were statistically processed using the MetaboAnalyst 5.0 online software (29). The multivariate analysis included principal component analysis (PCA), partial least square discriminant analysis (PLSDA), heatmaps and random forest analysis. Significant differences $(p \leq 0.05)$ between mean values were evaluated by one-way ANOVA. The oneway ANOVA statistics also included the post-hoc Tukey HSD test (made by MetaboAnalyst 5.0 algorithm) for a good statistical evaluation of differences between oil samples for each type of phytochemical (see Supplementary Files). 


\section{Results}

\subsection{UV-VIS Spectral Fingerprinting Related to Botanical Origin}

The individual UV-VIS spectra of all oils were recorded and analyzed as a first criteria of oil recognition based on the spectral fingerprint and absorbance intensity (data not shown). The sunflower (SFO) and walnut (WO) oils showed absorbances mainly in the UV region (220-280, $325 \mathrm{~nm}$ and 220-280 nm, respectively). The pumpkin oil (PO) group was recognized by VIS intense peaks at 450, 526, 568, 590 and $620 \mathrm{~nm}$, indicating their carotenoid, acylated anthocyanin and chlorophyll content. The spectra of linseed (LSO) and soybean (SO) oils showed higher absorbance at 448 and $443 \mathrm{~nm}$, respectively, as indicators of quinones content. The hempseed $(\mathrm{HO})$ and olive (EVOO) oil groups were characterized by strong absorbance at $668 \mathrm{~nm}$ (indicator of chlorophylls). The sea buckthorn (SBO) oils had a unique fingerprint with highest absorbance at $445-470 \mathrm{~nm}$, indicating a rich carotenoid content. These spectra offered a first preliminary information about the specific fingerprints of each oil category and a rough indicator of authenticity [25].

\subsection{GC-MS Profile, Univariate Analysis and Multivariate Classification of Oils Based on Fatty Acids Composition}

Based on the GC-MS analysis (typical chromatograms of each oil category are presented in Figure S2), the percentages of individual fatty acids (FAs) in all oils were determined, then the average values and standard deviations were calculated for commercial oils and compared to the authentic ones. Table S1 includes the individual fatty acid levels expressed in \% of area of the total identified fatty acid areas in the GC-MS chromatograms of oils under investigation. These raw data were processed by one-way ANOVA including the Tukey HSD post-hoc test (Table S2) to evaluate the significance of differences between the oil groups, considering each of the 10 fatty acids separated and identified by GC-MS. The graphic representations of the Tukey HSD statistical differentiations (made by the MetaboAnalyst 5.0 algorithm) are also included in Table S2. The specific unique composition of SBO is clearly observed, significantly different from other oils due to fatty acids C16:1n-7, C18:1n-7, C16:0, and C18:2n-6. The linseed oil (LSO) was significantly different due to the presence of $\mathrm{C} 18: 3 \mathrm{n}-3$, the hempseed oil $(\mathrm{HO})$ was differentiated due to its higher content in C18:3n-6 and C20:0, while sunflower oils (SFO) had a significant, higher content of C22:0. Olive oils were differentiated by their higher content of C18:1n-9. Stearic acid (C18:0) was present in all studied oils at different levels and cannot be considered specific, although soybean showed the highest content.

The multivariate analysis was applied for complementary information and included PCA (data not shown) and PLSDA. Figure 1 included the PLSDA scores plot and the heatmap graph which evidentiates the specific fingerprints of these oil category (Figure 1).

The PLSDA score plot (Figure 1a) shows a co-variance of $45.6 \%$ and indicates a good discrimination between groups, and especially between SBO vs. SFO and EVOO. Based on PLSDA loadings, the FAs with higher VIP scores were C20:0 and C18:3n-6 ( $\gamma$-linolenic acid) as markers for $\mathrm{HO}, \mathrm{C} 18: 1 n-9$ for EVOO, C18:2n-6 for WO, C22:0 for SFO, C18:3n-3 for LSO oils. Figure $1 \mathrm{~b}$ illustrates the heatmap of specific fatty acids characterizing each individual sample. There were identified increased C18:0 levels for commercial SO3 and PO2 samples and C22:0 for SFO1 and SFO2 samples. These levels can be considered potential indicators of adulteration, but such data need further confirmation.

Table 2 presents comparatively the mean values and standard deviations $(x \pm S D)$ registered for the main classes of fatty acids, namely saturated (SFA), monounsaturated (MUFA) and polyunsaturated (PUFA) fatty acids identified in all eight types of oils. Samples SFO, PO, HO, SO and WO showed the highest percentages of C18:2n-6 (58 to 81\% of total FAs), while LSO had maximum values of $52-67 \%$ of C18:3n-3. In SBO, palmitic acid was a major FA (C16:0 at 35.8-36.2\%), while in the EVOO group of oils, oleic acid (C18:1n-9) had values from $76.5 \%$ to $82.9 \%$. Differences were noticed also between the percentages of the other mono- or polyunsaturated acids. 


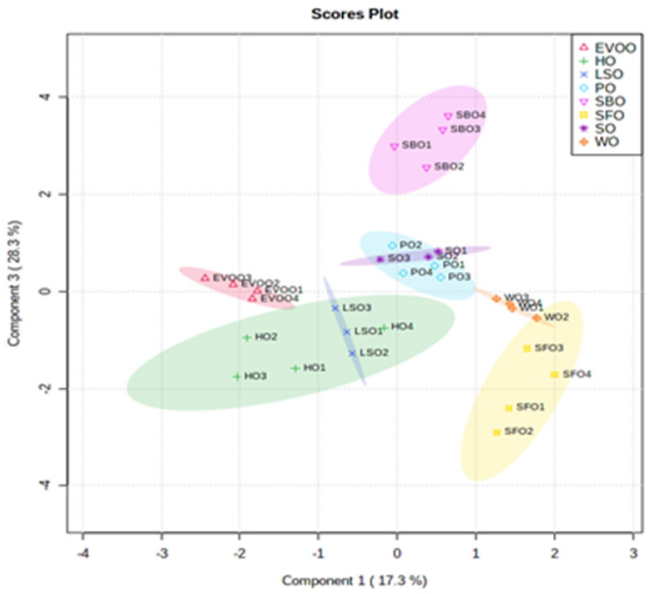

(a)

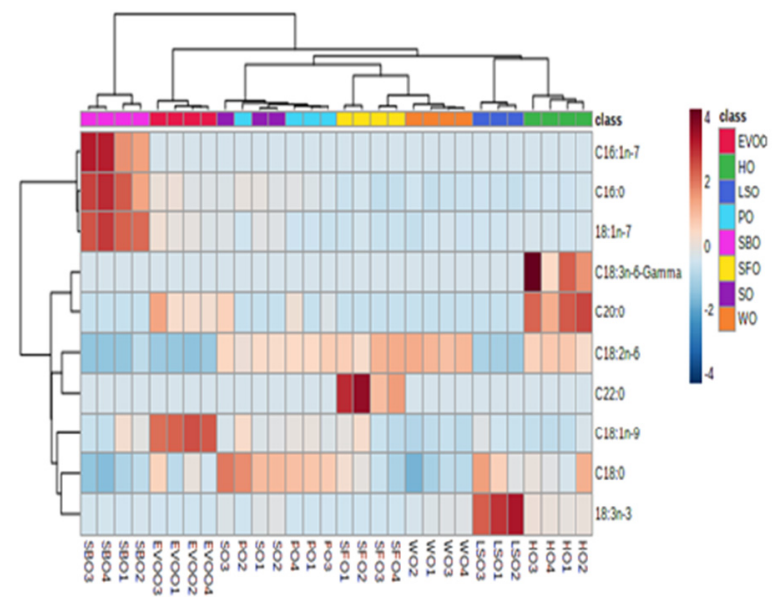

(b)

Figure 1. (a) The PLSDA score plot for the discriminatory analysis of the eight classes of oils. (b) The heatmap illustrating the individual fatty acids to be considered potential biomarkers of each oil group.

Table 2. Mean values and standard deviations $(x \pm S D)$ representing the percentages of saturated (SFA), monounsaturated (MUFA), polyunsaturated (PUFA) fatty acids identified in each type of oil. The ratios PUFA/MUFA and MUFA + PUFA/SFA (unsaturation index) are presented in columns 5 and 6 , respectively. For oil abbreviations, see Table 1.

\begin{tabular}{cccccc}
\hline Oil Type & SFA $(\mathbf{x} \pm \mathbf{S D})$ & MUFA $(\mathbf{x} \pm \mathbf{S D})$ & PUFA $(\mathbf{x} \pm \mathbf{S D})$ & PUFA/MUFA & MUFA + PUFA/SFA \\
\hline SFO & $5.621 \pm 1.855$ & $20.396 \pm 11.349$ & $73.982 \pm 13.175$ & 3.627 & 16.791 \\
PO & $11.042 \pm 2.125$ & $27.659 \pm 6.014$ & $61.297 \pm 8.052$ & 2.216 & 13.569 \\
LSO & $6.556 \pm 1.657$ & $17.510 \pm 4.691$ & $75.933 \pm 6.247$ & 4.337 & 14.253 \\
HO & $7.614 \pm 1.277$ & $13.833 \pm 4.175$ & $78.551 \pm 5.409$ & 5.678 & 12.133 \\
SO & $11.865 \pm 0.754$ & $21.891 \pm 2.232$ & $66.243 \pm 1.863$ & 3.026 & 7.428 \\
WO & $4.405 \pm 0.075$ & $9.949 \pm 3.311$ & $85.645 \pm 3.360$ & 8.608 & 21.700 \\
SBO & $36.783 \pm 7.364$ & $52.171 \pm 4.251$ & $11.045 \pm 1.559$ & 0.212 & 1.719 \\
EVOO1,3 & $13.251 \pm 2.183$ & $78.306 \pm 2.284$ & $8.443 \pm 1.053$ & 0.107 & 6.546 \\
EVOO2,4 & $10.166 \pm 1.568$ & $85.879 \pm 3.068$ & $3.955 \pm 0.869$ & 0.046 & 8.836 \\
\hline
\end{tabular}

The ratios PUFA/MUFA and MUFA + PUFA/SFA (considered as an unsaturation index) of each category can discriminate roughly between highly unsaturated ones (WO) and medium unsaturated oils (SFO, HO, LSO, PO, SO, EVOO). The SBO group of oils was represented mainly by palmitic $\mathrm{C} 16: 0$ and palmitoleic $\mathrm{C} 16: 1$ acid and showed the lowest unsaturation index, the PUFA content being mainly represented by C18:2n- 6 (5.2-11.4\%). The $\mathrm{SO}$ and EVOO groups were similar in unsaturation index, but $\mathrm{SO}$ had a higher percentage of PUFAs due to C18:2n-6 and C18:1n-9, compared to EVOO. The highest ratio PUFA/MUFA and unsaturation was noticed for WO, represented mainly by C18:2n- 6 and C18:1n-9 (Figure 1b). The HO and LSO oils contained distinctly C18:3n-3 at 52.5-67.1\% and $9.9-10.1 \%$ respectively, the first one having a superior unsaturation. The unsaturation of SFO and PO (represented mainly by C18:2n-6 and C18:1n-9 at percentages higher than $85 \%$ ) was found to be higher compared to SO and EVOO.

No significant differences were noticed between the authentic samples and the commercial ones, when fatty acids were considered as potential biomarkers of authenticity. These data are in good agreement with recent publications which referred mainly to olive oils and sunflower, hemp, linseed (flaxseed), walnut and sea buckthorn oils [32-35]. For sunflower oils, recent data showed a dominant level of $64 \%$ linoleic acid C18:2n-6, while in our study the percentage reached an average of $70 \%$; for flaxseed oils linolenic acid represented $46.5 \%$, while in our case it was around $65 \%$, with similar percentages being noticed by other authors $[36,37]$. The levels of the fatty acids were useful for the detection of frauds of olive oil (rich in C18:1) with sunflower, soybean and walnut, even at levels of 
adulteration (below 5\%), as demonstrated previously [38]. Sunflower, soybean, linseed and olive oils were found to have different percentages of linolenic/linoleic/oleic or palmitic acid, dominated by palmitoleic acid in sea buckthorn oil, by oleic acid in olive oil, linoleic acid in sunflower and soybean [37], and linolenic in pumpkinseed oil $(46.40 \%)$, while in our samples the percentage reached $60 \%$. The characterization of walnut oils was recently published [39,40] and showed levels of $62-74 \%$ C18:2 and 10\% C18:3. In our study, the level of C18:2n-6 reached $80 \%$ and $5 \% \mathrm{C} 18: 3 n-3$. Fewer data were available for hempseed oils, e.g., $55 \% \mathrm{C} 18: 2,16 \% \mathrm{C} 18: 3$ and $11 \% \mathrm{C} 18: 1$ [41]; our data showed mean values of 70\% C18:2n-6, $12 \%$ C18:1, 10\% C18:3n-3, and also 1\% C18:3n-6. A special FA composition was noticed for sea buckthorn oils, in our study this oil being characterized by approx. $35 \% \mathrm{C} 16: 0$, followed by approx. $25 \% \mathrm{C} 18: 1-9,24 \% \mathrm{C} 16: 1 \mathrm{n}-7,8 \% \mathrm{C} 18: 2-6$, and specifically by $5 \% \mathrm{C} 18: 1-7$, a good marker of recognition. Similar composition was noticed by other authors [42], e.g., 30-33\% C16:0, 30-35\% C16:1,14-18\% C18:1, 5-7\% C18:2, and up to 30\% C18:3.

\subsection{ITEX/GC-MS Profile of Volatiles, Univariate and Multivariate Analysis}

Table S3 presents the individual composition of volatile oils' components expressed in \% of peak area of the total volatiles, as determined by ITEX/GC-MS (mean values of duplicated separations). For oil abbreviations, see Table 1.

The univariate one-way ANOVA including Tukey's HSD post-hoc test was done (Table S4 includes the graphic representations of significant differences) to evaluate the significance of differences between the oil groups, considering the volatile molecules separated and identified by ITEX/GC-MS. The ethyl ester of hexanoic acid, heptanal, betapinene and beta-cis ocimene, 3 methyl butanoic acid and the ethyl ester of octanoic acid evidentiate as specific biomarkers of SBO, significantly different from other oils. Meanwhile, alpha-ocimene and acetophenone can be considered biomarkers of SFO, while olive oils were specifically recognized by their higher levels of hexyl acetate, 2,7 dimethyl 1-octanol and 3-hexenol acetate. Benzoic acid was a common component of many oils, with SFO being the richest source of it.

Figure 2a represents the multivariate data analysis, including the PLSDA score plot with a good discrimination between the different individual oils and oil groups (co-variance of $44.4 \%$ ), considering all volatile molecules separated and identified. Figure $2 \mathrm{~b}$ includes the heatmap which illustrates the differences between individual samples and oil categories.

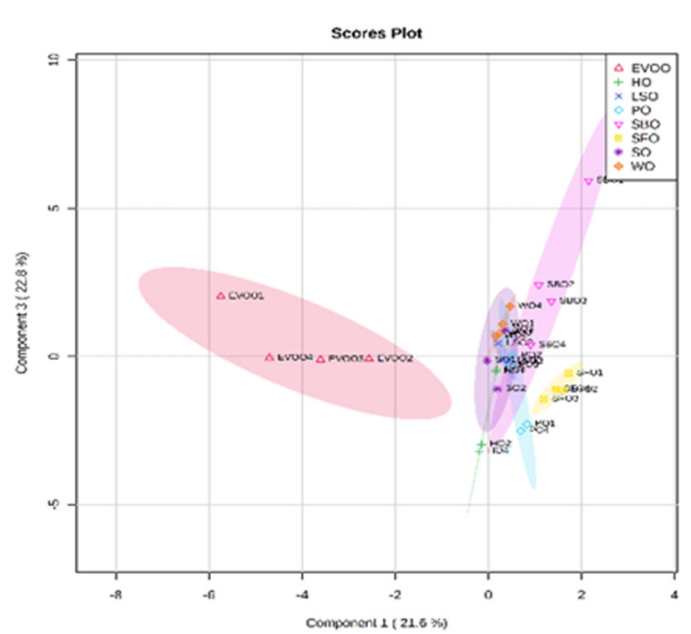

(a)

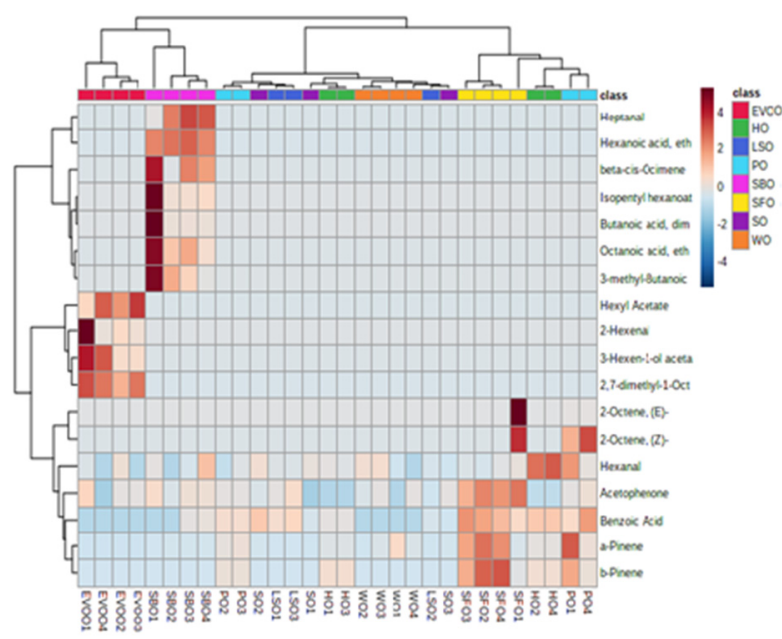

(b)

Figure 2. (a) PLSDA score plot for the discriminatory analysis of the eight classes of oils, based on ITEX/GC-MS analysis of volatiles. (b) Heatmap illustrating the volatile biomarkers of each individual oil.

The VIP scores calculated from PLSDA analysis classify the most relevant 15 molecules which may explain the discrimination between samples (data not shown). The highest 
values were recorded for dimethyl octane, hexyl acetate, hexenol and hexenal, all in the EVOO group. Other distinct groups of molecules (hexanoic acid, heptanal, ocimene, octanoic acid and methyl butane, isopentyl hexane) were specifically representing the SBO. We identified specific volatiles in EVOO samples, mainly hexenal, hexenol, hexyl acetate, and 2,7 dimethyl octanol, while for PO, LSO, HO and WO, mainly a-pinene and hexanal were found at different proportions, as shown in Figure 3b. The other molecules (pinenes, hexanal, 2-octene) were less specific but in different levels in the oils' groups. Comparing the authentic samples with the commercial ones, distinct differences were identified for sample EVOO1 in the EVOO group, for SBO1 (the genuine sample) comparing with the other samples in the SBO group, and for SFO1 samples in the SFO group. Such differences are possible indicators of adulteration. Different profiles were noticed for SFO, represented by a- and b-pinene, 2-octene and acetophenone, and for $\mathrm{SBO}$ recognized by the presence of butanoic acid derivatives, isopentyl hexanoate and b-cis-ocimene, responsible for the specific flavor of this oil. Few data were reported about volatiles responsible for the sensorial characteristics of these oils, but generally they were similar, mainly for olive oils [43-45], but also for linseed [36] and walnut [39,40].

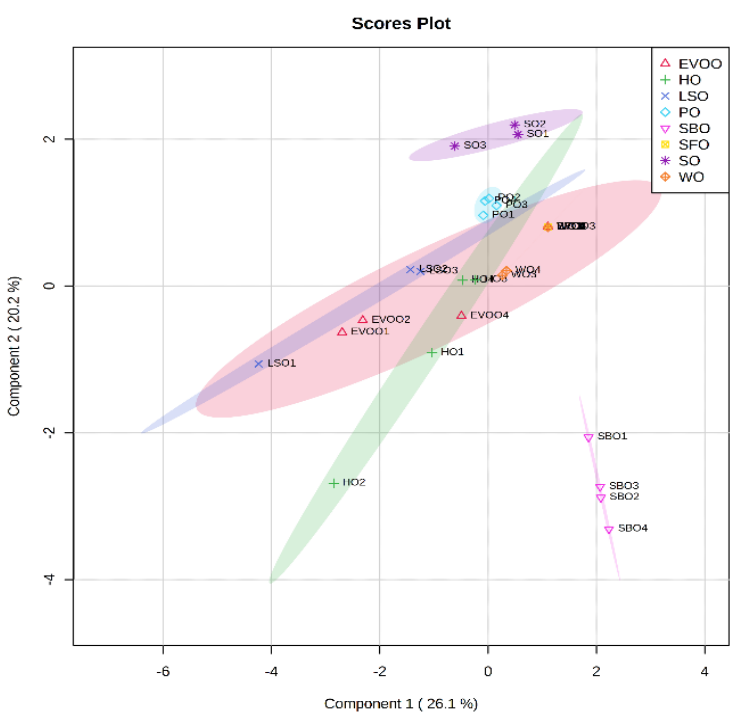

(a)

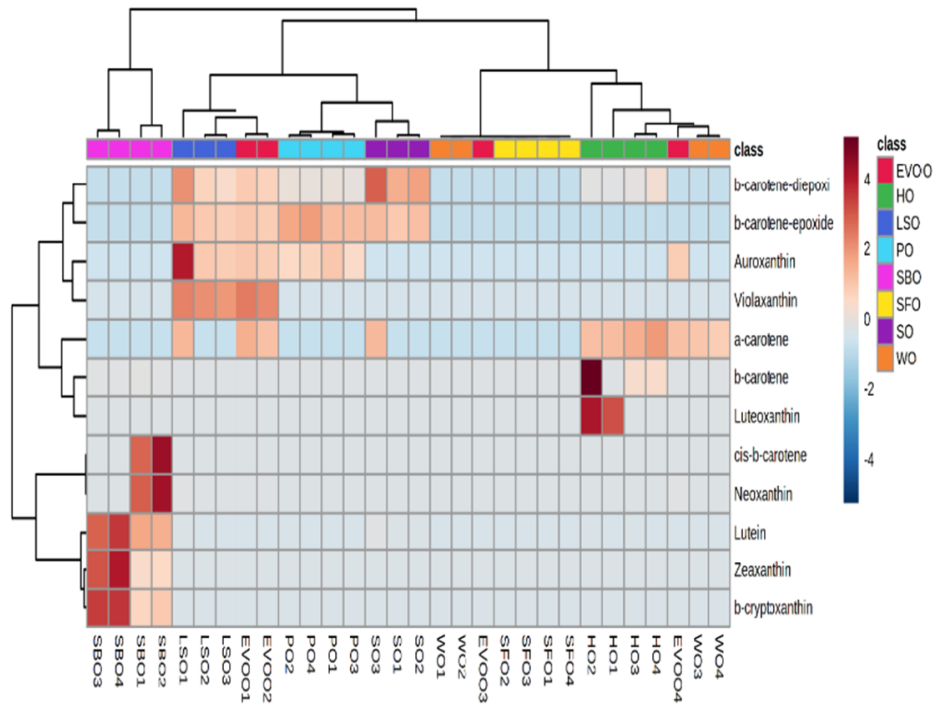

(b)

Figure 3. (a) PLSDA score plot for the discriminatory analysis of the eight classes of oils, considering their carotenoid contents. (b) Heatmap representing the Euclidian distance using Ward algorithm to identify the similarities between individual oils.

\subsection{HPLC-DAD Separation, Identification and Quantitation of Carotenoids}

Table 3 represents the mean concentrations of carotenoids found in commercial SFO, $\mathrm{PO}, \mathrm{LSO}, \mathrm{HO}, \mathrm{SO}, \mathrm{WO}, \mathrm{EVOO}$ and $\mathrm{SBO}$ oils, comparative to authentic oils from each category. The retention time and the absorption spectra determined by diode array detection (DAD) were useful for the identification of individual molecules, while the peak intensity values recorded from the UV-VIS spectra were used to calculate the concentration.

Concerning the total carotenoid content, a large range of concentrations were noticed, from mean values of $0.092 \mathrm{mg} / 100 \mathrm{~g}$ in SFO, to $0.15 \mathrm{mg} / 100 \mathrm{~g}$ in WO, 0.452 and $0.257 \mathrm{mg} / 100 \mathrm{~g}$ in LSO and PO, respectively, $0.366-0.395 \mathrm{mg} / 100 \mathrm{~g}$ in EVOO, $0.471-0.906 \mathrm{mg} / 100 \mathrm{~g}$ in $\mathrm{SO}$, and $49.470-129.751 \mathrm{mg} / 100 \mathrm{~g}$ in SBO. SBO was, by far, the richest source of these pigments. The univariate one-way ANOVA including Tukey's HSD post-hoc test (Table S5 includes the graphic representations of significant differences) reflected the significance of differences between the oil groups, considering carotenoids; SBO was, by far, the richest source of $\beta$-carotene, lutein, zeaxanthin and $\beta$-cryptoxanthin, while PO was the richest source of $\beta$-carotene epoxide, and $\mathrm{SO}$ a source of high $\beta$-carotene diepoxide. Luteoxanthin 
was found especially in hempseed and olive oils, while violaxanthin and auroxanthin were in LSO.

Table 3. Mean values $(\mathrm{mg} / 100 \mathrm{~g})$ of individual carotenoids separated and identified in commercial and authentic oils, respectively. For oil abbreviations, see Table 1.

\begin{tabular}{|c|c|c|c|c|c|c|c|c|c|c|c|}
\hline $\begin{array}{l}\text { Mean Values } \\
\quad \pm \text { SD }\end{array}$ & LUT & ZEA & $\alpha-\mathrm{CAR}$ & $\beta$-CAR & $\begin{array}{c}\beta \text {-CAR- } \\
\text { Diepoxide }\end{array}$ & $\begin{array}{l}\beta \text {-CAR- } \\
\text { Epoxide }\end{array}$ & $\beta$-CRP & ARO & NEO & VLX & LUX \\
\hline SFO1-3 & 0.038 & 0.036 & 0 & 0.018 & 0 & 0 & 0 & 0 & 0 & 0 & 0 \\
\hline SD & 0.001 & 0.001 & 0 & 0.001 & & & & & & & \\
\hline aSFO4 & 0.039 & 0.038 & 0 & 0.019 & 0 & 0 & 0 & 0 & 0 & 0 & 0 \\
\hline PO1-3 & 0.156 & 0.042 & 0 & 0.027 & 0.023 & 0.022 & 0 & 0.032 & 0 & 0 & 0 \\
\hline SD & 0.053 & 0.001 & & 0.003 & 0.002 & 0.002 & & 0.005 & & & \\
\hline $\mathrm{aPO} 4$ & 0.134 & 0.043 & 0 & 0.026 & 0.022 & 0.027 & 0 & 0.02 & 0 & 0 & 0 \\
\hline LSO1-2 & 0.245 & 0.055 & 0.021 & 0.024 & 0.055 & 0.020 & 0 & 0.069 & 0.059 & 0.038 & 0 \\
\hline SD & 0.239 & 0.021 & 0.001 & 0.005 & 0.022 & 0.002 & 0 & 0.044 & 0.030 & 0.002 & 0 \\
\hline aLSO3 & 0.065 & 0.040 & 0 & 0.020 & 0.033 & 0.018 & 0 & 0.036 & 0.034 & 0.035 & 0 \\
\hline HO1-3 & 0.196 & 0.040 & 0.020 & 0.041 & 0.019 & 0 & 0 & 0 & 0 & 0 & 0.098 \\
\hline SD & 0.080 & 0.001 & 0.002 & 0.001 & 0.001 & & & & & & \\
\hline $\mathrm{aHO} 4$ & 0.168 & 0.031 & 0.025 & 0063 & 0.028 & 0 & 0 & 0 & 0 & 0 & 0.0077 \\
\hline $\mathrm{SO} 2-3$ & 0.798 & 0.064 & 0.010 & 0.028 & 0.076 & 0.021 & 0.016 & 0 & 0 & 0 & 0 \\
\hline SD & 0.358 & 0.016 & 0.014 & 0.006 & 0.018 & 0.001 & 0.022 & & & & \\
\hline aSO1 & 0.396 & 0.043 & 0.000 & 0.022 & 0.057 & 0.019 & 0.000 & 0 & 0 & 0 & 0 \\
\hline WO1-3 & 0.046 & 0.045 & 0.018 & 0.022 & 0 & 0 & 0.034 & 0 & 0 & 0 & 0 \\
\hline SD & 0.002 & 0.008 & 0.001 & 0.001 & & & 0.001 & & & & \\
\hline aWO4 & 0.044 & 0.037 & 0.017 & 0.022 & 0 & 0 & 0.035 & 0 & 0 & 0 & 0 \\
\hline SBO2-4 & 16.000 & 94.640 & 0 & 5.077 & 0 & 0 & 10.218 & 0 & 3.816 & 0 & 0 \\
\hline SD & 5.411 & 58.011 & & 1.354 & & & 4.984 & & 0.987 & & \\
\hline aSBO1 & 10.903 & 25.422 & 0 & 7.037 & 0 & 0 & 3.431 & 0 & 2.677 & & 0 \\
\hline EVOO2,4 & 0.093 & 0.041 & 0.011 & 7.037 & 0.044 & 0.019 & 0.031 & 0.038 & 0.037 & 0.041 & 0.085 \\
\hline SD & 0.082 & 0.009 & 0.015 & 0.020 & 0 & 0 & 0 & 0 & 0 & 0 & 0 \\
\hline EVOO1,3 & 0.130 & 0.042 & 0.019 & 0.027 & 0.040 & 0.018 & 0.030 & 0.036 & 0.054 & 0.038 & 0.076 \\
\hline SD & 0.009 & 0.001 & 0.001 & 0.001 & 0 & 0 & 0 & 0 & 0.027 & 0 & 0.002 \\
\hline
\end{tabular}

ZEA, zeaxanthin; BC, $\beta$-carotene; $\beta$-CRP, $\beta$-cryptoxanthin; ARO, auroxanthin; $N E O$, neoxanthin; LUX, luteoxanthin; $\mathrm{CAR}$, total carotenoids; TOC, total tocopherols.

Significant differences between the authentic and commercial samples were noticed for LSO, SO, SBO and between extravirgin $(\mathrm{EVOO} 1,3)$ and virgin $(\mathrm{EVOO} 2,4)$ olive oil subgroups, in all cases lutein and zeaxanthin being responsible for such differences.

Figure 3a represents the PLSDA score plot for the discriminatory analysis considering their carotenoid contents. The heatmap illustrates the similarities between oil samples inside a group and among groups (Figure $3 b$ ).

The univariate and multivariate analysis confirm the specificity of carotenoid composition for each oil category, as mentioned above. The PLSDA score plot shows in this case a co-variance of $46.3 \%$ for the first two components, with good discrimination between SBO and SO oils; the other oil groups showing less differentiation. The VIP scores shows the most significant 15 molecules to differentiate oils: SBO due to $\beta$-carotene, lutein, zeaxanthin, $\beta$-cryptoxanthin, while luteoxanthin, $\beta$-carotene and $\alpha$-carotene were specifically representing HO. Meanwhile violaxanthin and auroxanthin were specific to LSO. Comparing the authentic samples with the commercial ones (Figure 3b), the heatmap shows identified distinct differences for sample EVOO3 in the EVOO group, for $\mathrm{HO} 1$ and $\mathrm{HO} 2$ comparative to the authentic sample HO4. Different concentrations of carotenoids, highly dependent on the botanical and geographical origin of vegetables, were reported in some recent publications $[41,46]$ and are in agreement with our data. 


\subsection{HPLC-RF Separation, Identification and Quantitation of Tocopherol Isomers}

For tocopherols' quantitation, the HPLC separation with fluorescence (RF) detection allowed the registration of the peak intensities of each type of the four isomers $(\alpha, \beta, \gamma, \delta)$, comparative to pure standards, as presented in Table 4.

Table 4. Mean values (mg/100 g) of individual tocopherol isomers separated and identified in commercial and authentic oils. For oil abbreviations, see Table 1.

\begin{tabular}{ccccc}
\hline Mean Values \pm SD & $\alpha$-Tocopherol & $\beta$-Tocopherol & $\gamma$-Tocopherol & $\delta$-Tocopherol \\
\hline SFO1-3 & 50.955 & 2.257 & 4.887 & 0.000 \\
SD & 0.290 & 2.285 & 3.779 & 0.000 \\
aSFO4 & 55.340 & 2.560 & 1.670 & 0.000 \\
\hline PO1-3 & 6.833 & 6.540 & 32.203 & 2.393 \\
SD & 4.923 & 10.375 & 8.394 & 1.315 \\
aPO4 & 11.670 & 3.450 & 32.340 & 2.930 \\
\hline LSO1-2 & 0.835 & 6.375 & 12.270 & 0.385 \\
SD & 0.672 & 1.025 & 4.059 & 0.021 \\
aLSO3 & 1.970 & 6.650 & 13.660 & 0.980 \\
\hline HO1-3 & 4.213 & 2.507 & 35.877 & 1.783 \\
SD & 1.592 & 1.020 & 6.855 & 0.524 \\
aHO4 & 4.430 & 2.890 & 36.440 & 1.980 \\
\hline SO2-3 & 7.725 & 0.000 & 57.955 & 36.390 \\
SD & 1.633 & 0.000 & 2.496 & 9.433 \\
aSO1 & 13.410 & 0.000 & 60.740 & 19.910 \\
\hline WO1-3 & 3.700 & 1.287 & 18.343 & 4.220 \\
SD & 1.854 & 1.118 & 4.148 & 0.944 \\
aWO4 & 3.220 & 1.660 & 17.550 & 3.980 \\
\hline SBO2-4 & 73.943 & 29.207 & 2.887 & 0.000 \\
SD & 33.263 & 18.390 & 1.636 & 0.000 \\
aSBO1 & 23.400 & 42.250 & 42.690 & 0.000 \\
\hline EVOO1,3 & 82.885 & 3.210 & 6.935 & 2.060 \\
SD & 105.366 & 1.089 & 7.969 & 1.923 \\
\hline EVOO2,4 & 154.135 & 10.500 & 13.800 & 0.000 \\
SD & 17.487 & 3.422 & 3.437 & 0.000 \\
\hline & & & &
\end{tabular}

The total concentration of tocopherols (TOC) ranged from $12.52 \mathrm{mg} / 100 \mathrm{~g}$ (commercial PO1-3) to $178.435 \mathrm{mg} / 100 \mathrm{~g}$ in EVOO2,4. Besides EVOO, the SBO, SO and SFO oils proved to be oils rich in tocopherols. Concomitantly, variations of the four tocopherol isomer levels were noticed in some groups. The SFO oils were characterized by high $\alpha$-tocopherols, while $\mathrm{SO}$ was rich in $\gamma$ and $\delta$-tocopherols. In the SBO group, the authentic sample had a 1:1 ratio of $\beta$ - and $\gamma$-tocopherols, as major isomers, while the commercial samples showed decreased concentrations.

The univariate one-way ANOVA including Tukey's HSD post-hoc test (Table S6 including the graphic representations of significant differences) reflected the significance of differences between the oil groups, considering the different tocopherol isomers: $\alpha$ tocopherol was dominant in olive oils EVOO and to a lesser extent in SBO, $\beta$-tocopherol had the highest level in SBO, and $\delta$-tocopherol was dominant in SO samples. Meanwhile in all oils $\beta$-tocopherol was commonly found in different concentrations. The PLSDA score plot shows in this case a co-variance of $61.1 \%$ for the first two components, with good discrimination between EVOO, SBO and SO oils.

Figure 4 shows the PLSDA score plot for the discriminatory analysis considering their tocopherol contents, and also the heatmap illustrating the specificity of individual oils and oil categories. 


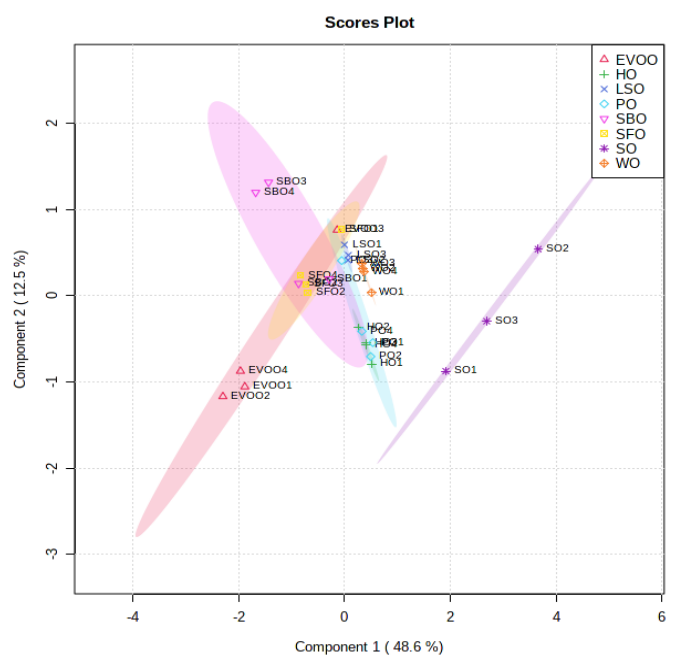

(a)

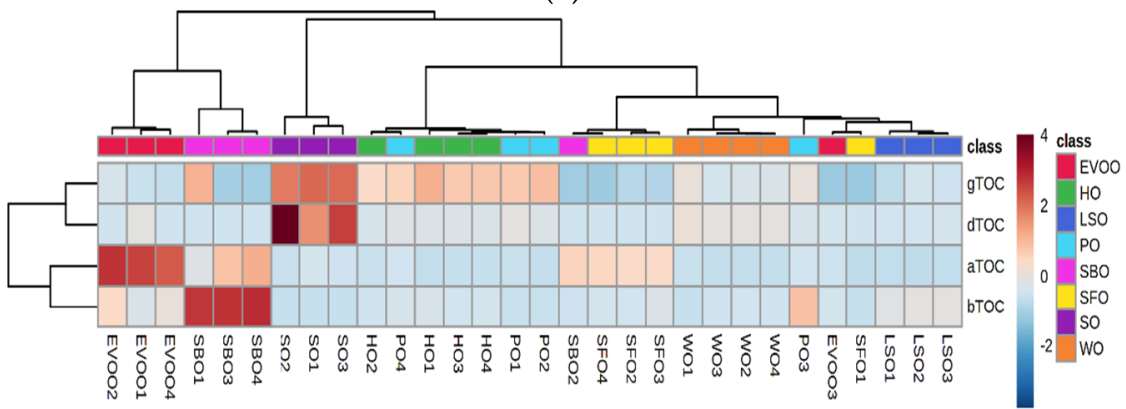

(b)

Figure 4. (a) PLSDA score plot for the discriminatory analysis of the eight classes of oils, considering their tocopherol contents. (b) Heatmap representing the Euclidian distance using the Ward algorithm to identify the similarities between individual oils.

\subsection{LC-ESI $-M S, H P L C-D A D$ and Spectrometric Evaluation of Individual and Total Phenolic Derivatives}

For phenolic derivatives, by the LC-ESI+-MS analysis, the separation and identification of polyphenols was done, based on the retention times, peak areas and the $m / z$ values for parental ions and fragments. In parallel, based on the calibration curves (Figure S1) of pure standards using the HPLC-DAD system, the retention times and peak areas were considered, and the concentration of individual compounds was determined. Concomitantly, the fast spectrophotometric FC method was applied for the quantitative evaluation and compared with the data obtained by HPLC-DAD. Table S7 includes the phenolic composition of individual oils, expressed in $\mathrm{mg} / 100 \mathrm{~mL}$ oil according to the calibrations made for each category of phenolics, as presented in Figure S1 (Supplementary File). These data were obtained by HPLC-DAD (TP-LC) and compared with the values obtained by the spectrometric method.

A large variety of phenolic derivatives were separated and identified in all samples, specific to each oil category, e.g., hydroxy benzoic and hydroxycinnamic acids (gallic, protocatechuic, vanilic, elagic, syringic and cafeic, coumaric, synapic, dicaffeoyl quinic, chlorogenic acids, respectively), flavonoids (quercetin, isorhamnetin, luteolin, gallocatechin, daidzein, genistein), as well as lignans (pino- and matairesinols), tyrosols, oleuropein and juglone.

The EVOO, SBO and SO were classified as "high-phenolic" oils compared to SFO, LSO, PO, HO, and WO. The TP values determined by HPLC-DAD were around three times superior to the values obtained by spectrometry (TP-FC), a result which is obvious considering that the FC method is based on the reducing capacity of free hydroxyl groups of 
phenolics' and is not an accurate determination of all phenolic derivatives. As noticed also by other authors [47], we found high statistical correlation $(p<0.005)$ between TP-HPLC and TP-FC values. Meanwhile, one should be considered that lignans and flavonoids do not show any linear relationship with FC data, suggesting caution about interpretation of FC results for oils having very different phenolic profiles.

Based on LC-ESI+-MS analysis and individual peak areas for each phenolic derivative, the multivariate analysis by PLSDA score plot shows a co-variance of $33.1 \%$ for the first two components, with good discrimination between EVOO, SBO and HO oils, the other oil groups showing less differentiation (Figure 5a). Figure $5 \mathrm{~b}$ represents the heatmap which illustrates the specific composition of each oil category, according to their phenolic composition.

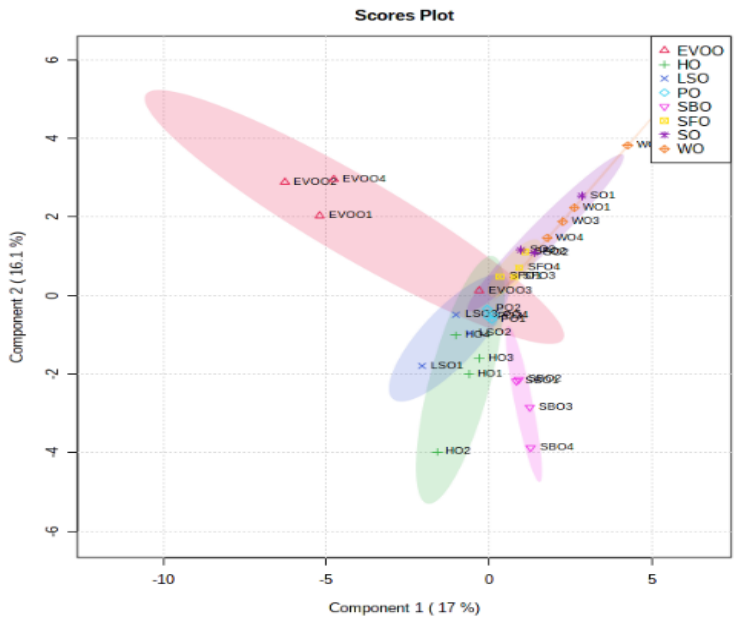

(a)

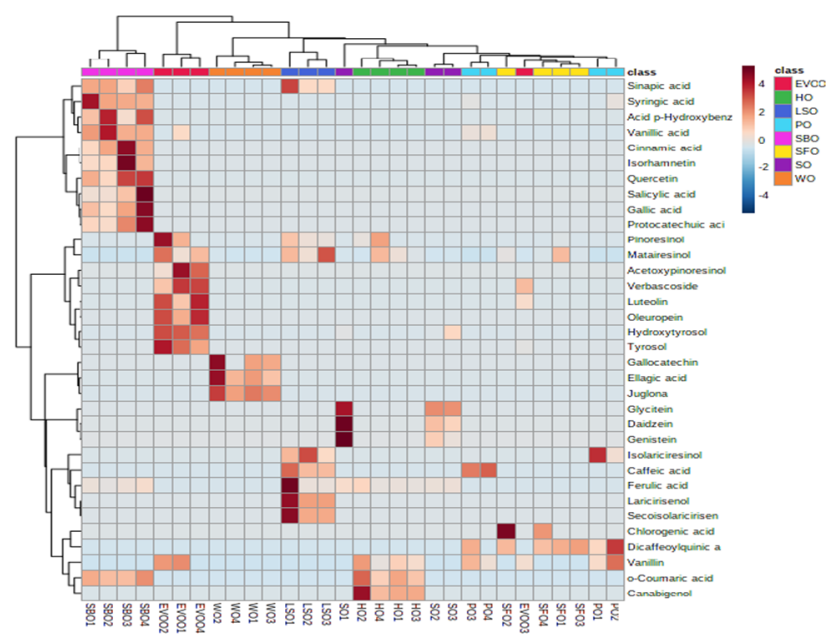

(b)

Figure 5. (a) PLSDA score plot for the discriminatory analysis of the eight categories of oils, considering their content in phenolic derivatives. (b) Heatmap representing the Euclidian distance, and by the Ward algorithm, the similarities between individual oil samples inside a group and among groups.

Based on PLSDA analysis, the VIP scores showed the most significant 15 molecules to differentiate these oils. Therefore, SFO was specifically characterized by chlorogenic and dicaffeoylquinic acids, PO by vanillin and caffeoylquinic acid, LSO by matairesinol and pinoresinol, $\mathrm{HO}$ by canabigenol, $\mathrm{SO}$ by glycitein, daidzein and genistein, WO by juglone, ellagic acid and gallocatechin, EVOO by oleuropein, tyrosol and hydroxytyrosol, luteolin, pino-, matai- and acetoxipinoresinols, and SBO by many phenolic acids from hydroxybenzoic and hydroxycinnamic subclasses. Intuitive images are given by heatmaps of individual oils (Figure 5b), phenolics being good biomarkers to discriminate qualitatively and quantitatively the individual oil samples, as well the different categories of oils.

\section{Discussion}

The large variability regarding the composition of edible vegetable oils is reflected by many reports and publications, as presented above. Therefore, it is very difficult to compare data released from different European regions, even for the same type of oils, since the botanical variety of the oil raw sources (seeds or fruits), the geographic location and environment conditions, the fractions used to produce the oil (seeds or pulp or whole fruit) and the technological parameters are strongly influencing their final composition.

The data presented here reflected mainly the composition of oils manufactured in Romania, at low scale (the authentic ones) or industrial scale. Their composition did not differ significantly, although the commercial samples had some quantitative differences for the five phytochemical categories. 
The data obtained by HPLC-DAD-RF-MS and GC-MS, complemented with statistical multivariate analysis, brings added value for the discrimination, classification, and authentication of these edible oils. These results are complementary and in good agreement with our previous studies using the ATR-FTIR spectrometry coupled with chemometrics [25].

The univariate analysis using one-way ANOVA and Tukey's HSD post-hoc test combined with multivariate analysis showed significant discriminations among oils used in this study, either their botanical authentication, or their individual variations due to processing parameters.

The profiles of fatty acids (Figure 1a) show similarities between SO and PO oils, represented mainly by C18:0. LSO is represented mainly by C18:3n-3, while WO by C18:2n-6 and EVOOs by C18:1n-9. Specifically, SFO was identified by C22:0 and C18:2n-6, while HO by C18:3n-6 ( $\gamma$-linoleic acid) and C20:0, and finally, SBO by C16:0, C16:1n-7 and C18:1n-9. Our data were compared to other publications, as presented in Section 3.2, the average levels of fatty acids being similar, in spite of their different origins.

For volatiles, the heatmap shows a good discrimination between the profiles of the eight oil groups, especially between EVOO, SFO, SBO. Stronger discriminations were observed between SFO (rich in 2-octene, acetophenone, $\alpha$ - and $\beta$-pinenes), for EVOOs rich in hexyl derivatives (hexenol, hexenal, hexyl acetate), and for SBO, rich in volatile acids (butanoic, hexanoic, butanoic), heptanal and ocimen. WO, LSO and SO showed less significant biomarkers, while $\mathrm{HO}$ was represented by a higher content of hexanal and $\mathrm{PO}$ by octene and pinenes. Few data were reported about volatiles responsible for the sensorial characteristics of these oils, but generally they were similar, mainly for olive oils [43-45], but also for linseeds [36] and walnut [39,40].

Beside the relevance of volatiles for the oil origin and identity, one can also consider the influence of the thermal processing on volatiles' elaboration, their different sensorial characteristics being due not only to the raw material, but also to technological parameters (temperature-time and storage conditions).

Concerning carotenoid pigments, $\mathrm{HO}$ was the richest source of $\beta$ - and $\alpha$-carotene and luteoxanthin, while SO was the richest in $\beta$-carotene epoxides. The LSO was identified easily by a high content in auroxanthin and violaxanthin, beside $\beta$-carotene epoxides. The samples EVOO had lower levels of auroxanthin, violaxanthin and $\alpha$-carotene' By far, the richest content of carotenoids, especially zeaxanthin, lutein, $\beta$-carotene and $\beta$ cryptoxanthin was reported in SBO, followed by WO, EVOO, and in lower amounts in SO. Different concentrations of carotenoids, highly dependent on the botanical and geographical origin of vegetables were reported in some recent publications [41,46] and are in agreement with our previous and present data.

Significant differences were observed in relation to the different tocopherol isomers. While $\alpha$-tocopherol was found as major molecule in EVOO, SBO and SFO, $\beta$-tocopherol was mostly representative for SBO, $\gamma$ - and $\delta$-tocopherol for SO. This intuitive map also helps to make a ranking of these oils according to the relative content of carotenoids and tocopherols, useful for their recognition, e.g., SBO, HO and SO with specific, different compositions, as well EVOO, PO and LSO groups having distinct groups of molecules which may be considered as biomarkers. Relevant recent articles found similar or different levels for tocopherols in similar vegetable oils [35,37,40,41,48-52].

The most significant discrimination between the oils was obtained considering the phenolic derivatives, as a mirror of their identity. The richest oils in phenolics were EVOO and SBO, represented specifically by tyrosols, oleuropein, verbascoside, luteolin and pinoresinols, and by quercetin and isorhamnetin together with many phenolic acids (gallic, syringic, vanilic, protocatecuic, cinnamic, salycilic), respectively. Then, LSO is recognized specifically by lignans (pino-, matairesinols larici, isolarici and secoisolarici resinols), WO by juglone, elagic acid and gallocatechin, and SO by daidzein, glycitein, and genistein. HO has its typical phenolics, canabigenol, o-coumaric acid and vanillin, while PO is characterized by dicaffeoyl quinic acid and vanillin. Finally, SFO is the richest source of chlorogenic acid and dicaffeoyl quinic acid. Similar phenolics, having different 
ranges, were also mentioned by different publications, especially the ones related to olive oils [45,53,54], but also for linseed [37], walnut [40], or sea buckthorn [48] oils.

\section{Conclusions}

The data presented here recommend a systematic analytical flow based on a metabolomic approach to be applied for edible oils, but also for other plant-based foods, or byproducts, identifying the profile of valuable components, including phytochemicals, as authenticity biomarkers and health-promoting molecules.

The results of our previous and current investigations demonstrated that a succession of instrumental analysis combined with chemometric models, such as PLSDA and heatmap clustering, have a good predictive ability to detect the oils' botanical origin, as well as to identify qualitative and quantitative differences between individual samples from the same phenotype and the specific features of each category of oils. Therefore, these complementary data are useful not only to identify the oil category but also the quality of commercial oils (which may be mislabeled or adulterated), comparative to the authentic ones of the same botanical and geographical origin.

Finally, we are recommending the data fusion of such complementary investigations to have a more clear and accurate evaluation and identity of commercial edible oils, and especially of functional oils (cold pressed, virgin, extravirgin) richer in phytochemicals as bioactive molecules.

This study needs validation for an extended number of samples (authentic and commercial) to have a more accurate image of the phytochemical biomarkers to be considered in an "identity" card of each type of edible oil, including their quality determined by specific processing.

Supplementary Materials: The following supporting information can be downloaded at: https: / / www.mdpi.com/article/10.3390/app12041933/s1. Figure S1: Calibration curves made by HPLC$\mathrm{DAD}$, with different pure standards of phenolic derivatives: gallic acid and vanillin (to quantify hydroxybenzoic acid derivatives), clorogenic and ferulic acid (for hydroxicinnamic derivatives), quercetin (for flavonoids), luteolin (for flavones), oleuropein (for tyrosols), and pinoresinol (for lignans). Figure S2: Typical GC-MS chromatograms of the different oils under investigation. For oils' abbreviations, see Table 1. Table S1: Individual fatty acid levels expressed in \% of area of the total identified fatty acid areas in the GC-MS chromatograms of oils under investigation (mean values of duplicated separations). For oils' abbreviations, see Table 1. Table S2: Results of one-way ANOVA and Tukey's HSD post-hoc test to evaluate the significance of differences between the oil groups, considering each of the 10 fatty acids separated and identified by GC-MS. The graphic representations of the Tukey HSD (made by MetaboAnalyst 5.0 algorithm) are presented below. Table S3: Individual composition of volatile oils' components expressed in \% of peak area of the total volatiles, as determined by ITEX/GC-MS (mean values of duplicated separations). For oils' abbreviations, see Table 1. Table S4: Results of one-way ANOVA and Tukey's HSD post-hoc test to evaluate the significance of differences between the oil groups, considering all volatiles separated and identified by ITEX GC-MS. The graphic representations of the Tukey HSD (made by MetaboAnalyst 5.0 algorithm) are presented below. Table S5: Results of one-way ANOVA and Tukey's HSD posthoc test to evaluate the significance of differences between the oil groups, considering carotenoid molecules separated and identified by HPLC-DAD. The graphic representations of the Tukey HSD are attached below. Table S6: Results of one-way ANOVA and Tukey's HSD post-hoc test to evaluate the significance of differences between the oil groups, considering the tocopherol isomers identified by HPLC-FD. The graphic representations of the Tukey HSD are attached below. Table S7: The phenolic composition of all oil samples: mean values expressed in $\mathrm{mg} / 100 \mathrm{~mL}$ oil and standard deviations (SD), as determined by HPLC-DAD. For details, see Section 2.

Author Contributions: Conceptualization, C.S.; methodology, F.D., S.S., F.F. and F.R.; software, C.S.; validation, A.B., S.S. and F.D.; formal analysis, F.D., S.S., F.F. and F.R.; writing-original draft preparation, C.S., F.R. and F.F.; writing-review and editing, C.S. and A.P.; supervision, project administration and funding acquisition, C.S. and A.P. All authors have read and agreed to the published version of the manuscript. 
Funding: This research was funded by the Executive Unit for Financing Higher Education, Research, Development, and Innovation (UEFISCDI) within the framework of project PN-III-P1-1.2-PCCDI2017-0046/No. 1/2018, within PNCDI III and from the National Research Development Projects to finance excellence (PFE)-14/2022-2024 granted by the Romanian Ministry of Research and Innovation.

Institutional Review Board Statement: Not applicable.

Informed Consent Statement: Not applicable.

Data Availability Statement: Not applicable.

Acknowledgments: The authors acknowledge the donations of authentic oil samples from different manufacturers.

Conflicts of Interest: The authors declare no conflict of interest.

\section{References}

1. Gunstone, F. (Ed.) Vegetable Oils in Food Technology: Composition, Properties and Uses, 2nd ed.; Wiley Blackwell: Hoboken, NJ, USA, 2011; 376p, ISBN 978-1-444-33991-8.

2. Salah, W.A.; Nofal, M. Review of some adulteration detection techniques of edible oils. J. Sci. Food Agric. 2020, 101, 811-819. [CrossRef] [PubMed]

3. Dubois, V.; Breton, S.; Linder, M.; Fanni, J.; Parmentier, M. Fatty acid profiles of 80 vegetable oils with regard to their nutritional potential. Eur. J. Lipid Sci. Technol. 2007, 109, 710-732. [CrossRef]

4. Hernandez, E.M. Specialty Oils: Functional and Nutraceutical Properties. In Functional Dietary Lipids, Food Formulation, Consumer Issues and Innovation for Health; Woodhead Publisher for Series in Food Science, Technology and Nutrition; Woodhead Publishing: London, UK, 2016; pp. 69-101.

5. Socaciu, C.; Dulf, F.; Ranga, F.; Fetea, F.; Bele, C.; Echim, C. Selected biomarkers and methods to fingerprint vegetable functional oils originating from Romania. In Proceedings of the Conference Euro Food Chem XV-Food for the Future, Copenhagen, Denmark, 5-8 July 2009; pp. 162-165.

6. Socaciu, C.; Ranga, F.; Fetea, F.; Leopold, D.; Dulf, F.; Parlog, R. Complementary advanced techniques applied for plant and food Authentication. Czech Food Sci. 2009, 27, S70-S75. [CrossRef]

7. Bajoub, A.; Bendini, A.; Fernández-Gutiérrez, F.; Carrasco-Pancorbo, A. Olive oil authentication: A comparative analysis of regulatory frameworks with especial emphasis on quality and authenticity indices, and recent analytical techniques developed for their assessment A review. Crit. Rev. Food Sci. Nutr. 2018, 58, 832-857. [CrossRef] [PubMed]

8. Lioupi, A.; Nenadis, N.; Theodoridis, G. Virgin olive oil metabolomics: A review. J. Chromatogr. B 2020, 1150, 122-161. [CrossRef] [PubMed]

9. Messai, H.; Farman, M.; Sarraj-Laabidi, A.; Hammami-Semmar, A.; Semmar, N. Chemometrics Methods for Specificity, Authenticity and Traceability Analysis of Olive Oils: Principles, Classifications and Applications. Foods 2016, 5, 77. [CrossRef] [PubMed]

10. Da Ros, A.; Masuero, D.; Riccadonna, S.; Brkić Bubola, K.; Mulinacci, N.; Mattivi, F.; Lukić, I.; Vrhovsek, U. Complementary Untargeted and Targeted Metabolomics for Differentiation of Extra Virgin Olive Oils of Different Origin of Purchase Based on Volatile and Phenolic Composition and Sensory Quality. Molecules 2019, 24, 2896. [CrossRef]

11. Servili, M.; Montedoro, G. Contribution of phenolic compounds to virgin olive oil quality. Eur. J. Lipid Sci. Technol. 2002, 104, 602-613. [CrossRef]

12. Olmo-García, L.; Carrasco-Pancorbo, A. Chromatography-MS based metabolomics applied to the study of virgin olive oil bioactive compounds: Characterization studies, agro-technological investigations and assessment of healthy properties. TrAC Trends Anal. Chem. 2021, 135, 116153. [CrossRef]

13. Boskou, D. Phenolic Compounds in Olives and Olive Oil. In Olive Oil: Minor Constituents and Health, 1st ed.; Boskou, D., Ed.; CRC Press: Boca Raton, FL, USA, 2009; Chapter 3; pp. 11-44.

14. Lerma Garcia, M.J. Characterization and Authentication of Olive and Other Vegetable Oils; Springer: Berlin/Heidelberg, Germany, 2012.

15. Wang, L.; Chen, Y.; Ye, Z.; Hellmann, B.; Xu, X.; Jin, Z.; Ma, Q.; Yang, N.; Wu, F.; Jin, Y. Screening of Phenolic Antioxidants in Edible Oils by HPTLC-DPPH Assay and MS Confirmation. Food Anal. Methods 2018, 11, 3170-3178. [CrossRef]

16. Li, C.; Yao, Y.; Zhao, G.; Cheng, W.; Liu, H.; Liu, C.; Shi, Z.; Chen, Y.; Wang, S. Comparison and analysis of fatty acids, sterols, and tocopherols in eight vegetable oils. J. Agric. Food Chem. 2011, 59, 12493-12498. [CrossRef] [PubMed]

17. Zhang, L.; Li, P.; Sun, X.; Wang, X.; Xu, B.; Wang, X.; Ma, F.; Zhang, Q.; Ding, X. Classification and adulteration detection of vegetable oils based on fatty acid profiles. J. Agric. Food Chem. 2014, 62, 8745-8751. [CrossRef] [PubMed]

18. Shen, M.; Zhao, S.; Zhang, F.; Huang, M.; Xie, J. Characterization and authentication of olive, camellia and other vegetable oils by combination of chromatographic and chemometric techniques: Role of fatty acids, tocopherols, sterols and squalene. Eur. Food Res. Technol. 2021, 247, 411-426. [CrossRef]

19. Mota, M.F.S.; Waktola, H.D.; Nolvachai, Y.; Marriott, P.J. Gas chromatography-mass spectrometry for characterization, assessment of quality and authentication of seed and vegetable oils. TrAC Trends Anal. Chem. 2021, 138, 116238. [CrossRef] 
20. Bagur-González, M.G.; Pérez-Castaño, E.; Sánchez-Viñas, M.; Gázquez-Evangelista, D. Using the liquid chromatographic fingerprint of sterols fraction to discriminate virgin olive from other edible oils. J. Chromatogr. A 2015, 1380, 64-70. [CrossRef] [PubMed]

21. Yuan, C.; Xie, Y.; Jin, R.; Ren, L.; Zhou, L.; Zhu, M.; Ju, Y. Simultaneous analysis of tocopherols, phytosterols, and squalene in vegetable oils by high-performance liquid chromatography. Food Anal. Methods 2017, 10, 3716-3722. [CrossRef]

22. Esteki, M.; Simal-Gandara, J.; Shahsavari, Z.; Zandbaaf, S.; Dashtaki, E.; Heyden, Y.V. A review on the application of chromatographic methods, coupled to chemometrics, for food authentication. Food Control 2018, 93, 165-182. [CrossRef]

23. Li, X.; Kong, W.; Shi, W.; Shen, Q.A. A combination of chemometric methods and GC-MS for the classification of edible vegetable oils. Chemometr. Intell. Lab. Syst. 2016, 155, 145-150. [CrossRef]

24. Biancolillo, A.; Marini, F.; Ruckebusch, C.; Vitale, R. Chemometric Strategies for Spectroscopy-Based Food Authentication. Appl. Sci. 2020, 10, 6544. [CrossRef]

25. Socaciu, C.; Fetea, F.; Ranga, F.; Bunea, A.; Dulf, F.; Socaci, S.; Pintea, A. Attenuated Total Reflectance-Fourier Transform Infrared Spectroscopy ATR-FTIR. Coupled with Chemometrics, to Control the Botanical Authenticity and Quality of Cold-Pressed Functional Oils Commercialized in Romania. Appl. Sci. 2020, 10, 8695. [CrossRef]

26. Giacomelli, L.M.; Mattea, M.; Ceballos, C.D. Analysis and characterization of edible oils by chemometric methods. J. Am. Oil Chem. Soc. 2006, 83, 303-308. [CrossRef]

27. Gómez-Caravaca, A.M.; Maggio, R.M.; Cerretani, L. Chemometric Applications to Assess Quality and Critical Parameters of Virgin and Extra-Virgin Olive Oil A Review. Anal. Chim. Acta 2016, 913, 1-21. [CrossRef] [PubMed]

28. Godoy, A.C.; Dos Santos, P.D.S.; Nakano, A.Y.; Bini, R.A.; Siepmann, D.A.B.; Schneider, R.; Gaspar, P.A.; Pfrimer, P.W.D.; da Paz R.F.; Santos, O.O. Analysis of Vegetable Oil from Different Suppliers by Chemometric Techniques to Ensure Correct Classification of Oil Sources to Deal with Counterfeiting. Food Anal. Methods 2020, 13, 1138-1147. [CrossRef]

29. Chong, J.; Wishart, D.S.; Xia, J. Using MetaboAnalyst 40 for Comprehensive and Integrative Metabolomics Data Analysis. Curr. Protoc. Bioinform. 2019, 68, e86. [CrossRef] [PubMed]

30. Tudor, C.; Gherasim, E.C.; Dulf, F.V.; Pintea, A. In vitro bioaccessibility of macular xanthophylls from commercial microalgal powders of Arthrospira platensis and Chlorella pyrenoidosa. Food Sci. Nutr. 2021, 9, 1896-1906. [CrossRef]

31. Singleton, V.L.; Orthofer, R.; Lamuela-Raventos, R.M. Analysis of total phenols and other oxidation substrates and antioxidants by means of the Folin-Ciocalteu reagent. Methods Enzymol. 1999, 299, 152-178.

32. Alves, A.Q.; da Silva, V.A.; Góes, A.J.S.; Silva, M.S.; de Oliveira, G.G.; Bastos, U.V.G.A.; de Castro Neto, A.G.; Alves, A.J. The Fatty Acid Composition of Vegetable Oils and Their Potential Use in Wound Care. Adv. Skin Wound Care 2019, 32, 1-8. [CrossRef]

33. Frančáková, H.; Ivanišová, E.; Dráb, S.; Krajčovič, T.; Tokár, M.; Mareček, J.; Musilová, J. Composition of Fatty Acids in Selected Vegetable Oils. Potravinarstvo 2015, 9, 538-542. [CrossRef]

34. Zielińska, A.; Nowak, I. Fatty acids in vegetable oils and their importance in cosmetic industry. Chem. Aust. 2014, 68, 103-110.

35. Kamal-Eldin, A.; Andersson, R. A multivariate study of the correlation between tocopherol content and fatty acid composition in vegetable oils. J. Am. Oil Chem. Soc. 1997, 74, 375-380. [CrossRef]

36. Krist, S.; Stuebiger, G.; Bail, S.; Unterweger, H. Analysis of volatile compounds and triacylglycerol composition of fatty seed oil gained from flax and false flax. Eur. Lipid Sci. Technol. J. 2006, 108, 48-60. [CrossRef]

37. Choo, W.S.; Birch, J.; Dufour, J.P. Physicochemical and quality characteristics of cold-pressed flaxseed oils. J. Food Comp. Anal. 2006, 20, 202-211. [CrossRef]

38. Christopoulou, E.; Lazaraki, M.; Komaitis, M.; Kaselimis, K. Effectiveness of determination of fatty acids and triglycerides for the detection of adulteration of olive oils with vegetable oils. Food Chem. 2004, 84, 463-474. [CrossRef]

39. Tsamouris, G.; Hatziantoniou, S.; Demetzos, C. Lipid analysis of Greek Walnut oil Juglans regia L. Z. Naturforsch. 2002, 57c, 51-56. [CrossRef]

40. Ojeda-Amador, R.M.; Desamparados Salvador, M.; Gómez-Alonso, S.; Fregapane, G. Characterization of virgin walnut oils and their residual cakes produced from different varieties. Food Res. Int. 2018, 108, 396-404. [CrossRef]

41. Montserrat-de la Paz, S.; Marín-Aguilar, F.; Garcia Gimenez, M.D.; Fernandez-Arche, M.A. Hemp (Cannabis sativa L.) seed oil: Analytical and phytochemical characterization of unsaponifiable fraction. J. Agric. Food Chem. 2014, 62, 1105-1110. [CrossRef]

42. Yang, B.; Kallio, H.P. Fatty acid composition of lipids in sea buckthorn (Hippophaë rhamnoides L.) berries of different origins. J. Agric. Food Chem. 2011, 49, 1939-1947. [CrossRef]

43. Diez-Simon, C.; Mumm, R.; Hall, R.D. Mass spectrometry-based metabolomics of volatiles as a new tool for understanding aroma and flavour chemistry in processed food products. Metabolomics 2019, 15, 41. [CrossRef]

44. Campestre, C.; Angelini, G.; Gasbarri, C.; Angerosa, F. The compounds responsible for the sensory profile in monovarietal virgin olive oils. Molecules 2017, 22, 1833. [CrossRef]

45. Lukić, I.; Lukić, M.; Žanetić, M.; Krapac, M.; Godena, S.; Brkić Bubola, K. Inter-Varietal Diversity of Typical Volatile and Phenolic Profiles of Croatian Extra Virgin Olive Oils as Revealed by GC-IT-MS and UPLC-DAD Analysis. Foods 2019, 8, 565. [CrossRef]

46. Tudor, C.; Bohn, T.; Iddir, M.; Dulf, F.V.; Focşan, M.; Rugină, D.O.; Pintea, A. Sea Buckthorn Oil as a Valuable Source of Bioaccessible Xanthophylls. Nutrients 2019, 12, 76. [CrossRef] [PubMed]

47. Alessandri, S.; Ieri, F.; Romani, A. Minor polar compounds in extra virgin olive oil: Correlation between HPLC-DAD-MS and the Folin-Ciocalteu spectrophotometric method. J. Agric. Food Chem. 2014, 62, 826-835. [CrossRef] [PubMed] 
48. Franke, S.; Frohlich, K.; Werner, S.; Bohm, V.; Schone, F. Analysis of carotenoids and vitamin E in selected oilseeds, press cakes and oils. Eur. J. Lipid Sci. Technol. 2010, 112, 1122-1129. [CrossRef]

49. Zielińska, A.; Nowak, I. Abundance of active ingredients in sea-buckthorn oil. Lipids Health Dis. 2017, 16, 95. [CrossRef]

50. Tyskiewicz, K.; Gieysztor, R.; Maziarczyk, I.; Hodurek, P.; Rój, E.; Skalicka-Wozniak, K. Supercritical Fluid Chromatography with Photodiode Array Detection in the Determination of Fat-Soluble Vitamins in Hemp Seed Oil and Waste Fish Oil. Molecules 2018, 23, 1131. [CrossRef]

51. Kallio, H.; Yang, B.; Peippo, P. Effects of Different Origins and Harvesting Time on Vitamin C, Tocopherols, and Tocotrienols in Sea Buckthorn (Hippophae rhamnoides. Berries. J. Agric. Food Chem. 2002, 50, 6136-6142. [CrossRef]

52. Schwartz, H.; Ollilainen, V.; Piironen, V.; Lampi, A.-M. Tocopherol, tocotrienol and plant sterol contents of vegetable oils and industrial fats. J. Food Comp. Anal. 2008, 21, 152-161. [CrossRef]

53. García, A.; Brenes, M.; García, P.; Romero, C.; Garrido, A. Phenolic content of commercial olive oils. Eur. Food Res. Technol. 2003, 216, 520-525. [CrossRef]

54. Klikarová, J.; Česlová, L.; Kalendová, P.; Dugo, P.; Mondello, L.; Cacciola, F. Evaluation of Italian extra virgin olive oils based on the phenolic compounds' composition using multivariate statistical methods. Eur. Food Res. Technol. 2020, 246, 1241-1249. [CrossRef] 